\title{
Proteome Analysis of Neural Stem Cells and Neurospheres infected with Zika Virus
}

\author{
Danielle G. Junqueira*, Stevens Rehen, Daniel Martins-de-Souza, Juliana M. Nascimento.
}

\begin{abstract}
The Brazilian outbreak of the Zika virus (ZIKV) in 2015 was accompanied by an increase in the number of cases of microcephaly, which involves severe changes in brain development, indicating a possible association between the viral infection with these cerebral malformations. The molecular mechanisms that promote these modifications have not been fully understood. In order to do so, in vitro models using human induced pluripotency stem cells (hiPSC), differentiated into neural cells, are being studied as a model of neurodevelopment. Here, we infected human neural progenitors (hNPCs) with ZIKV and dengue virus (DENV) strains. The infection was performed in the progenitor state and during its differentiation into neurospheres, in order to analyze in vitro the modifications caused by viral infection in these models. The samples, infected or not with one virus strain, underwent large-scale quantitative proteomic analysis, aiming to analyze differences and similarities of the effects of the Brazilian and African viral strains, as well as to compare ZIKV infection with DENV. The analysis of the cellular proteome allowed the identification of possible metabolic pathways and proteins, such as processing and splicing of mRNAs, Hippo, NIK/NF kappaB and Wnt signaling pathways. Which may be related to the mechanisms and development of the disease. And we were also able to point potential differences between the cellular response to the different virus strains analyzed.
\end{abstract}

\section{Key words:}

Infection, Neurodevelopment, IPSCs.

\section{Introduction}

With the spread of ZIKV by America in the years 2014 and 2015, there was an alarming mobilization due to the severe changes in brain development that were then related to this infectious disease ${ }^{1}$. Several studies, using both animal models and in vitro models, have sought answers in order to better understand the cellular targets and mechanisms of ZIKV in infected cells ${ }^{2}$. The ZIKV can promote changes in the nervous system due to its ability to invade neural tissue and its neurovirulence, being able to infect and proliferate in neuronal cells ${ }^{3}$.In this project, we performed the proteomic analysis of hNPCs and neurospheres infected with ZIKV and DENV strains. Aiming to investigate potential biochemical pathways associated with the mechanisms of the viruses, comparing the alterations observed in each virus strain.

\section{Results and Discussion}

The hNPCs and the neurospheres were infected with the Brazilian (ZIKV AB) and African (ZIKV 766) strains of the ZIKV, as wells as with DENV and maintained in culture for 3 days after infection. Then, the samples proceeded with the preparation for proteomic analyzes. In the analyses of the neurospheres samples, we identified and quantified 1727 proteins, with at least 1 unique peptide. Among these, 191 proteins were differentially regulated (Student's t-test $p<0.05$ ) in samples infected by ZIKV 766, 130 in samples infected with ZIKV AB, and 216 in the samples infected by DENV, all in comparison to control condition (MOCK). Regarding the analysis of the hNPCs, we identified 516 proteins differentially regulated in the samples infected by ZIKV $A B, 254$ in samples infected with ZIKV 766, and 109 in DENV-infected samples, all compared to MOCK. We performed the Pearson correlation of the samples, observed in Table 1, and it is possible to observe a higher correlation between ZIKV 766 and AB strains, than when these strains are correlated with the condition DENV. Performing the in silico analysis of the proteins identified in the hNPCs and neurospheres samples, we detected potential pathways associated with the infection mechanisms of the viruses and development of the disease, such as processing and splicing of mRNAs, Hippo signaling pathway, which is related to the control of cell proliferation, as well as changes in the NIK/NF kappaB and Wnt signaling pathways. Moreover, comparing the differently expressed proteins in each condition, we were able to investigate potential common and distinct cellular responses between the virus strains analyzed, such as proteins involved with translation initiation, ribosomal proteins and proteins related to metabolic pathways of catalysis and energy metabolism.

\begin{tabular}{|c|c|c|c|}
\hline & ZIKV 766 & ZIKV AB & DENV \\
\hline ZIKV 766 & 1 & & \\
\hline ZIKV AB & 0.436823 & 1 & \\
\hline DENV & 0.128199 & 0.310894 & 1 \\
\hline
\end{tabular}

Table 1. Pearson correlation between the conditions of ZIKV 766, $\mathrm{AB}$ and DENV.

\section{Conclusions}

Thus, in vitro model studies, associated with large-scale proteomic analysis, allows the identification of pathways and biological processes related to the changes caused by viral infection of ZIKV AB and 766, as well as DENV in neurodevelopment. Allowing a better understanding of viral mechanisms of action, and the search for drugs and therapeutic targets that inhibit the ability of ZIKV to infect neural progenitor cells. We identified some pathways associated with the different viruses, which could be further investigated to a better understanding of these mechanisms of viral infection and proliferation in neural progenitor cells.

\section{References}

${ }^{1}$ Gulland, A., (2016). Zika virus is a global public health emergency, declares WHO. BMJ (Clinical research ed.), 352(February), p.i657. ${ }^{2}$ Cugola, F.R. et al., (2016). The Brazilian Zika virus strain causes birth defects in experimental models. Nature, 534(7606), pp.267271. Dang, J. et al., 2016. Zika Virus Depletes Neural Progenitors in Human Cerebra Organoids through Activation of the Innate Immune Receptor TLR3. Cell Stem Cell, 19(2), pp. 258-265. ${ }^{3}$ Calvet, G. et al., 2016. Detection and sequencing of Zika virus from amniotic fluid of fetuses with microcephaly in Brazil: a case study. The Lancet Infectious Diseases, 16(6), pp.653-660. 